\title{
Exotic Cooling on Neutron Stars with Different Surface Compositions
}

\section{Tsuneo NODA*}

Department of Physics, Graduate school of Sciences, Kyushu University, Fukuoka 810-8560, Japan

E-mail: tsune@gemini.rc.kyushu-u.ac.jp

\section{Masa-aki HASHIMOTO}

Department of Physics, Faculty of Sciences, Kyushu University, Fukuoka 810-8560, Japan

E-mail: hashi@gemini.rc.kyushu-u.ac.jp

\section{Masayuki FUJIMOTO}

Department of Physics, Faculty of Sciences, Hokkaido University, Sapporo 060-0810, Japan E-mail: fujimoto@astro1.sci.hokudai.ac.jp

\begin{abstract}
The thermal evolution of isolated neutron stars depends on neutrino emission process, equation of state (EOS) of nuclear matter, and surface composition. Focusing on the neutrino emission process, we can classify the cooling models in two groups. First one is the "standard cooling model", which includes modified URCA process and bremsstrahlung process; these processes are believed to operate inside neutron stars. Others include some exotic processes at high density or high temperature, such as pion condensation or quark beta decay, which is named as "exotic cooling model". Since exotic models result in high neutrino emission, exotic neutron stars cool much faster than standard ones. We investigate the thermal evolution of isolated neutron stars, using both the standard and the exotic cooling processes, and two kinds of surface composition, $\mathrm{He}$ and $\mathrm{Fe}$. Furthermore we employ nucleon superfluidity models, and adopt a critical temperature as a density-independent parameter. We find that there exist parameter regions which can be consistent with the observational results.
\end{abstract}

International Symposium on Nuclear Astrophysics - Nuclei in the Cosmos - IX

June 25-30 2006

CERN, Geneva, Switzerland

${ }^{*}$ Speaker. 


\section{Introduction}

Hot and dense neutron star (NS) is born from the supernova explosion, and remains high temperature $\left(\sim 10^{10} \mathrm{~K}\right)$ and high density $\left(10^{14}-10^{15} \mathrm{~g} \mathrm{~cm}^{-3}\right)$ during few hours to days. If a neutron star is isolated, there is no heat source interior of the star: it cools down with time. If a neutron star is younger than about 1000 years old, The age of it can be determined from historical documents about "guest" stars, or by calculating the supernova remnant expansion rate. Therefore, if the effective temperature of the isolated neutron star is observed using the X-ray observatory satellite such as Chandra, the relation between the neutron star age and the effective temperature can be obtained [1].

Recently, Slane et al. analyzed the observational data of the pulsar PSR J0205+6449 in the supernova remnant 3C58 which is the remnant of SN1181, and showed the upper limit of the effective temperature $[2,3]$. The effective temperature of the pulsar is almost one half compared with the theoretical calculation with the standard cooling model. To explain this cool but young neutron star, the other strong cooling process is required. In the present paper, we choose pion condensation as an exotic cooling process, and present numerical results of neutron star cooling. They are compared with the observational data.

\section{Neutron Star Models}

\subsection{Basic Equations}

To calculate the neutron star cooling curve under the hydrostatic equilibrium, we use the stellar evolutionary code $[4,5]$. The spherical symmetric code includes general relativity. Basic equations can be written as [6]

$$
\begin{aligned}
\frac{d M_{\mathrm{tr}}}{d r} & =4 \pi r^{2} \rho_{\mathrm{t}} \\
\frac{d P}{d r} & =-G \frac{\rho_{\mathrm{t}} c^{2}+P}{r^{2} c^{2}}\left(M_{\mathrm{tr}}+\frac{4 \pi r^{3} P}{c^{2}}\right)\left(1-\frac{2 G M_{\mathrm{tr}}}{c^{2} r}\right)^{-1} \\
\frac{d \ln T}{d \ln P} & =\nabla_{\mathrm{rad}} \\
\frac{d M_{\mathrm{tr}}}{d M_{\mathrm{r}}} & =\frac{\rho_{\mathrm{t}}}{\rho}\left(1-\frac{2 G M_{\mathrm{tr}}}{c^{2} r}\right)^{1 / 2} \\
\frac{d \phi}{d M_{\mathrm{tr}}} & =\frac{G\left(M_{\mathrm{tr}}+\frac{4 \pi r^{3} P}{c^{2}}\right)}{4 \pi r^{4} \rho_{\mathrm{t}}}\left(1-\frac{2 G M_{\mathrm{tr}}}{c^{2} r}\right)^{-1} \\
\frac{d\left(L_{\mathrm{r}} e^{2 \phi / c^{2}}\right)}{d M_{\mathrm{r}}} & =e^{2 \phi / c^{2}}\left(\varepsilon_{\mathrm{n}}-\varepsilon_{v}-e^{-\phi / c^{2}} T \frac{\partial s}{\partial t}\right) .
\end{aligned}
$$

Here $M_{\mathrm{r}}$ and $M_{\mathrm{tr}}$ denote enclosed rest mass and gravitational mass within the radius $r . \rho$ and $\rho_{\mathrm{t}}$ are the rest mass density and mass energy density. $\varepsilon_{\mathrm{n}}$ and $\varepsilon_{v}$ describe energy gain and losses by the nuclear burning and by the neutrino emission. $\nabla_{\text {rad }}$ is the radiative gradient. $\phi$ is the gravitational potential, and $s$ is the entropy. 


\subsection{Equation of state}

We choose Lattimer \& Swesty equation of state (LSEOS) [7] for the high density region of $\rho>10^{8} \mathrm{~g} \mathrm{~cm}^{-3}$, and we connect it to BPS [8] for the low density region. For incompressibility, we select $K=180 \mathrm{MeV}$. Using this EOS, the maximum mass of NS is about $2.1 M_{\odot}$ and the radius is $10.9 \mathrm{~km}$. As seen in Figure 1, the mass and radius relation is consistent with an observational result of RXJ1856-3754 [9].

\subsection{Neutrino emission processes}

In the interior of NS, the mean free path of neutrinos is about $10^{5} \mathrm{~km}$, which is larger than the NS radius $(\sim 10 \mathrm{~km})$. Therefore neutrinos easily escape from the star without any interactions with the NS interior matter, and neutrinos carry internal heat energy outside from the star.

There are some commonly known neutrino emission processes. We consider two models of neutrino emission process, the standard cooling model and the exotic cooling model. The standard cooling model contains well-known modified URCA process and bremsstrahlung. The exotic cooling model includes some strong neutrino emission processes, which contains exotic particles. We adopt pion condensation URCA process [10] as the strong neutrino emission process. In the pion condensation theory, the condensation angle is written in terms of the Landau - Migdal (LM) parameter, $g^{\prime} \equiv \tilde{g}^{\prime}\left(f / m_{\pi}\right)^{2}$, which measures the correlation strength in the spin-isospin channel [11]. We adopt $\tilde{g}^{\prime}=0.5$ for our calculation. Also, there is the critical density where the pion phase appears, below which the phase of matter is normal nuclear matter. Therefore the pion condensation URCA process does not work below this limit.

We plot the density dependence of neutrino emission rates in Figure 2.

\subsection{Superfluidity}

In the interior of NS, nuclei are degenerate, and the temperature is low enough compared with the Fermi temperature $\left(T_{\mathrm{F}} \sim 10^{11} \mathrm{~K}\right)$. In this situation, when the temperature lows below a critical temperature $T_{\mathrm{c}}$, nuclei create Cooper pairs. Then pairs condense, and the matter transits to the superfluid state. Temperature below $T_{\mathrm{c}}$, neutrons transit to singlet state at low density $(\sim$ $10^{13-14} \mathrm{~g} \mathrm{~cm}^{-3}$ ) or to triplet state at high density $\left(\gtrsim 10^{14} \mathrm{~g} \mathrm{~cm}^{-3}\right)$, and protons transit into singlet superconductive state. In general, once in superfluid state, thermodynamical properties such as heat capacity decrease as a function of $\left(T_{\mathrm{c}} / T\right)$. Especially for neutrino emission, the rate changes proportional to $\exp \left(T_{\mathrm{c}} / T\right)$. However, because of theoretical uncertainty of nuclear forces, $T_{\mathrm{c}}$ has large uncertainty. $T_{\mathrm{c}}$ is thought in a range of $10^{7}-10^{10} \mathrm{~K}$ and as a function of $\rho$ [11]. To simplify a relation between cooling curves, we assume $T_{\mathrm{c}}$ as a density independent parameter and varies it in a range of $10^{7.8}-10^{8.3} \mathrm{~K}$.

\subsection{Photon emission processes}

After the supernova explosion, the dominant cooling process is the neutrino emission until about $10^{4} \mathrm{yr}$. Because of the temperature dependence of the emission rate, the photon emission overtakes neutrino emission for $t>10^{5} \mathrm{yr}$. Then the dominant cooling process becomes the photon emission. The surface compositions affect the cooling via photon because of the opacity differences. If the opacity of the surface composition is large (such as $\mathrm{Fe}$, or $\mathrm{Ni}$ ), the initial effective 
temperature of NS becomes lower, which leads to slow cooling in the photon emission dominated era. With small opacity, the effect is opposite. Usually, NS surface composition is thought to be ${ }^{56} \mathrm{Fe}$. However, considering accretion from the interstellar gas, colliding small objects or fallback of the supernova ejectile, the surface compositions are uncertain. The surface compositions of NS have not been well determined by observations. We adopt two extreme cases; ${ }^{4} \mathrm{He}$ for small opacity case and ${ }^{56} \mathrm{Fe}$ for large case.

\section{Results and discussion}

We have calculated neutron star cooling curves. We show the calculated results in Figure 3, and compare them with observed effective temperatures of isolated neutron stars [1, 2]in Figure 4 and Table 1. Using pion condensation URCA process as the typical exotic cooling, we have explained the effective temperature of PSR J0205+6449. Changing the surface composition and the superfluidity critical temperature as parameters, we find three domains which is consistent to effective temperatures of a neutron star, except 0822-4300. For 0822-4300, we could suppose that the mass is small as $M \lesssim 0.5 M_{\odot}$, and pion condensation does not exist even at the centre of the star.

\section{References}

[1] D. P. Page, http://www.astroscu.unam.mx/neutrones/NS-Data/data_good.dat (2002)

[2] P. O. Slane, D. J. Helfand \& S. S. Murray, New Constraints on Neutron Star Cooling from CHANDRA Observations of 3C 58 , Astrophys. J. Lett. 571 (2002) L45

[3] P. Slane, D. J. Helfand, E. Swaluw \& S. S. Murray, New Constraints on the Structure and Evolution of the Pulsar Wind Nebula 3C 58, Astrophys. J. 616 (2004) 403

[4] M. Y. Fujimoto, T. Hanawa, I. Iben Jr \& M. R. Richardson, Thermal Evolution of Accreting Neutron Stars, Astrophys. J. 278 (1984) 813

[5] T. Hanawa \& M. Y. Fujimoto, Thermal Response of Neutron Stars to Shell Flashes, Publ. Astron. Soc. Japan 36 (1984) 199

[6] K. S. Thorne, The Relativistic Equations of Stellar Structure and Evolution, Astrophys. J. 212 (1977) 825

[7] J. M. Lattimer \& F. D. Swesty, A Generalized Equation of State for Hot, Dense Matter, Nucl. Phys. A. 535 (1992) 331

[8] G. Baym, C. Pethick \& P. Sutherland, The Ground State of Matter at High Densities: Equation of State and Stellar Models , Astrophys. J. 170 (1971) 299

[9] T. M. Braje \& R. W. Romani , RX J1856-3754: Evidence for a Stiff Equation of State , Astrophys. J. $\mathbf{5 8 0}(2002) 1043$

[10] H. Umeda, K. Nomoto, S. Tsuruta, T. Muto \& T. Tatsumi , Neutron Star Cooling and Pion Condensation, Astrophys. J. 431 (1994) 309

[11] S. Tsuruta, Thermal Properties and Detectability of Neutron Stars. II. Thermal Evolution of Rotation-Powered Neutron Stars, Phys. Rep. 292 (1998) 1 

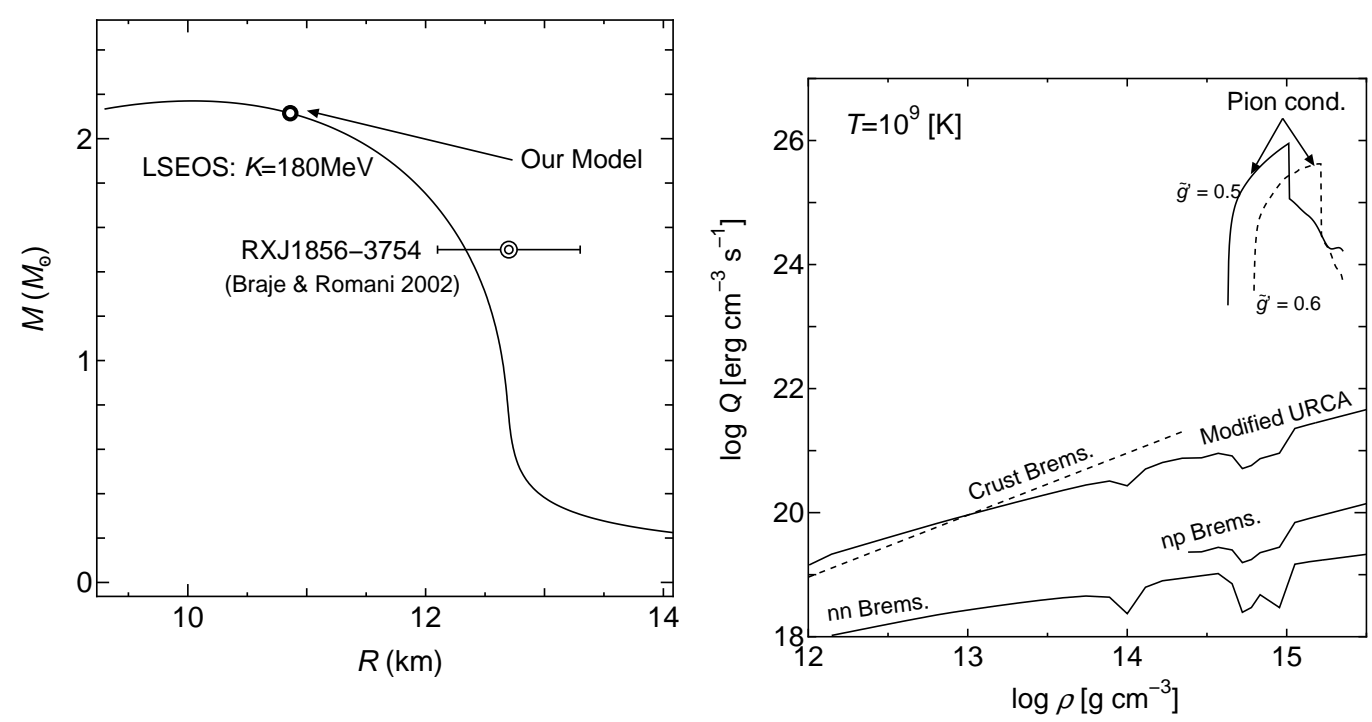

Figure 1: Mass - radius relation of neutron stars with LSEOS [7]. The circle on a line Figure 2: Density dependence of neutrino emisshows our model whose central density is $2.0 \times$ sion rates. The assumed coupling constant " $\tilde{g}^{\prime}=$ $10^{15} \mathrm{~g} \mathrm{~cm}^{-3}$. The double circle with the error bar 0.5 " indicates the pion condensation URCA proindicates the observational result of RXJ1856- cess for our model.

3754 [9].

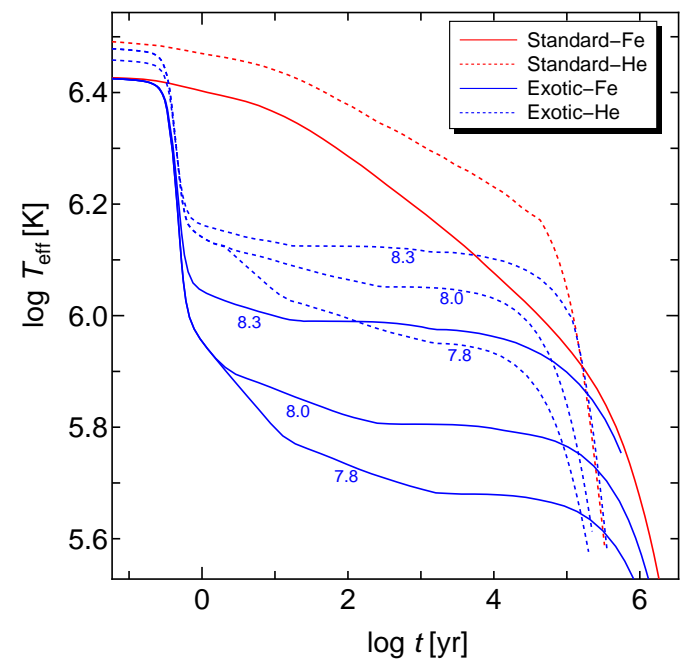

Figure 3: Calculated cooling curves of a neutron star. The Blue and the Red lines denote exotic cooling and standard cooling, respectively. Solid Blue and Green domains are regions which can and dotted lines show the difference due to the be explained by standard cooling, exotic cooling surface compositions, $\mathrm{Fe}$ and $\mathrm{He}$, respectively. and either cooling processes, respectively. ObNumbers below lines indicate the superfluidity servational results are taken from $[1,2]$. critical temperatures in Log-scale.

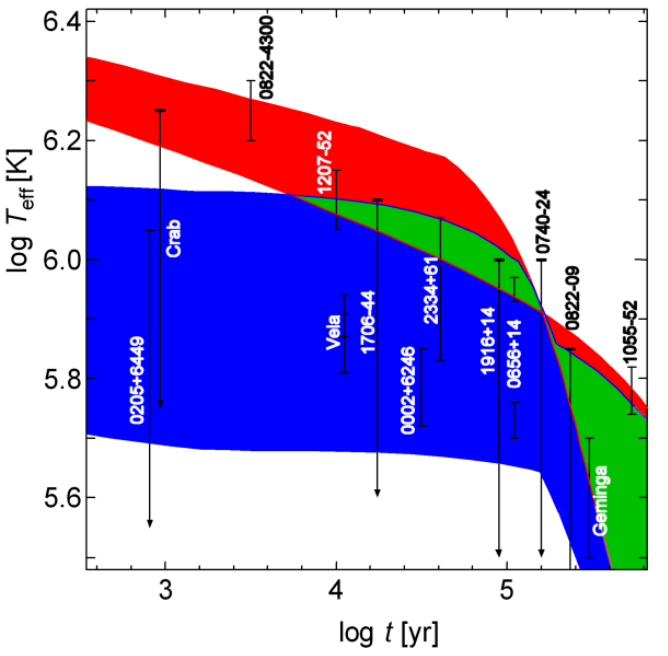

Figure 4: Comparison with observations. Red, 政 


\begin{tabular}{|c|c|c|c|c|c|}
\hline NS & $\begin{array}{l}\log t \\
(\mathrm{yr})\end{array}$ & & $\log T_{\mathrm{et}}$ & $(\mathrm{K})$ & Model \\
\hline $0205+6449$ & 2.91 & & & 6.05 & EXT \\
\hline Crab $(0531+21)$ & 2.97 & & & 6.25 & $\mathrm{STD}(\mathrm{Fe}), \mathrm{EXT}$ \\
\hline $0822-4300$ & 3.50 & 6.20 & - & 6.30 & $\mathrm{STD}(\mathrm{He})$ \\
\hline $1207-52$ & 4.00 & 6.05 & - & 6.15 & $\mathrm{STD}(\mathrm{Fe}), \mathrm{EXT}$ \\
\hline Vela (0833-45) & 4.05 & 5.81 & - & 5.94 & EXT \\
\hline $1706-44$ & 4.24 & & & 6.10 & $\mathrm{STD}(\mathrm{Fe}), \mathrm{EXT}$ \\
\hline $0002+6246$ & 4.50 & 5.72 & - & 5.85 & EXT \\
\hline $2334+61$ & 4.61 & 5.83 & - & 6.07 & $\mathrm{STD}(\mathrm{Fe}), \mathrm{EXT}$ \\
\hline $1916+14$ & 4.95 & & & 6.00 & STD(Fe), EXT \\
\hline \multirow[t]{2}{*}{$0656+14$} & 5.04 & 5.93 & - & 5.97 & $\mathrm{STD}(\mathrm{Fe}), \mathrm{EXT}$ \\
\hline & 5.04 & 5.70 & - & 5.76 & EXT \\
\hline $0740-28$ & 5.20 & & & 6.00 & $\mathrm{STD}(\mathrm{Fe} / \mathrm{He}), \mathrm{EXT}$ \\
\hline 1822-09 & 5.37 & & & 5.85 & $\mathrm{STD}(\mathrm{Fe}), \mathrm{EXT}$ \\
\hline Geminga $(0630+178)$ & 5.48 & 5.50 & - & 5.70 & $\mathrm{STD}(\mathrm{Fe}), \mathrm{EXT}$ \\
\hline $1055-52$ & 5.73 & 5.74 & - & 5.82 & $\mathrm{STD}(\mathrm{Fe}), \mathrm{EXT}$ \\
\hline
\end{tabular}

Table 1: Correspondence between observational results [1, 2] and cooling models. The column "Model" denotes the possible cooling models; "STD" means the standard and "EXT" means the exotic cooling models. For STD, possible surface compositions are displayed in blankets. For EXT, it is difficult to determine surface compositions uniquely. 